\title{
El juego como factor motivador en la enseñanza de la anatomía humana
}

\author{
Alberto García-Barrios, Ana I. Cisneros-Gimeno, M. José Luesma-Bartolomé, Jesús Benito-Rodríguez, \\ Eva Barrio-Ollero, Jaime Whyte-Orozco
}

Introducción. Con la llegada a la universidad de una nueva generación de alumnos, la 'generación Z', considerada como la primera generación nativa digital, se hace necesario implantar nuevas metodologías docentes en el ámbito universitario para mejorar el proceso de enseñanza-aprendizaje y conseguir una mayor motivación en el alumno. Para ello, el uso de nuevas tecnologías de la información y de la comunicación ha permitido dinamizar estos procesos y aumentar la motivación del alumnado en todos los niveles educativos, incluyendo la docencia universitaria.

Sujetos y métodos. Utilización de la aplicación educativa Kahoot a través de dispositivos móviles, con los alumnos matriculados de la asignatura de 'Anatomía Humana II (Esplacnología)', de segundo curso del Grado de Medicina en la Universidad de Zaragoza.

Resultados. El impacto que la experiencia ha causado en los estudiantes se ha medido a través de encuestas de valoración cualitativas, analizando sus resultados de aprendizaje. Estos resultados han sido valorados de manera muy positiva por el alumnado, tanto en lo referente a utilidad en la enseñanza-aprendizaje como a motivación.

Conclusión. Kahoot es una herramienta digital interactiva, gratuita y de manejo sencillo tanto para el docente como para los alumnos, que permite que éstos mejoren en su aprendizaje haciendo uso de nuevas tecnologías y se sientan así más motivados.

Palabras clave. Anatomía humana. Juego. Kahoot. Motivación.

\section{Game as a motivating factor in the teaching of human anatomy}

Introduction. The need to face a new generation of students, the ' $Z$ generation', considered as the first digital native generation, makes it necessary to implement new teaching methodologies in the university field to improve the teachinglearning process and above all achieve greater motivation in the student. To this end, the use of new information and communication technologies has enabled the dynamics of these processes and increasing student motivation at all educational levels, including university teaching.

Subjects and methods. Use of the Kahoot educational app was raised through mobile devices as an innovative tool that enhances motivation and learning process, participating students enrolled in the subject of 'Human Anatomy II (Esplacnology)', second course of the Degree of Medicine in the University of Zaragoza.

Results. The impact that the experience has caused students has been studied through qualitative assessment surveys, analyzing the learning outcomes obtained by students. The results achieved in terms of implementation of these new teaching-learning methodologies have been valued by students, in a very positive way both at the level of usefulness in learning teaching and at the level of motivation.

Conclusion. Kahoot is an interactive digital tool, free and easy to use for both teachers and students, and which allows the latter to improve their learning using new technologies, feeling more motivated.

Key words. Game. Human anatomy. Kahoot. Motivation.

\section{Introducción}

El Espacio Europeo de Educación Superior ha optado por una profunda renovación metodológica con el objetivo de conseguir una actualización de la oferta formativa por parte de las universidades es- pañolas [1]. Este nuevo marco educativo exige un esfuerzo por parte del profesorado, que debe innovar y mejorar sus metodologías y las herramientas para la docencia, con el fin de alcanzar y garantizar el aprendizaje del alumno por competencias (registrado en la guía docente de cada asignatura) des-
Departamento de Anatomía e Histología Humanas. Facultad de Medicina. Universidad de Zaragoza. Zaragoza, España.

Correspondencia: Dra. Ana Isabel Cisneros Gimeno. Departamento de Anatomía e Histología Humanas. Facultad de Medicina. Universidad de Zaragoza. Domingo Miral, s/n. E-50009 Zaragoza.

E-mail: aicisner@unizar.es 25.06.20.

Aceptado: 03.07.20.

Conflicto de intereses: No declarado.

Competing interests: None declared.

(c) 2020 FEM 
de la perspectiva de la innovación y eficacia didácticas. Uno de los aspectos más importantes para adecuarse a esta situación ha sido el impulso en la formación del equipo docente, entendiendo por tal un grupo de personas docentes con un mismo objetivo [2].

Hoy en día es importante desarrollar un perfil innovador en el aula universitaria, tanto desde el punto de vista de la calidad docente como de la necesidad de motivar al alumnado [3]. De hecho, en las aulas, nos enfrentamos al reto de enseñar a la 'generación Z' o posmilenial, alumnos nacidos entre los años 1995 y 2015, considerada como la primera generación nativa digital, lo que hace necesaria la incorporación de nuevas estrategias y mecanismos pedagógicos aplicados en el proceso de aprendizaje [4], utilizando la tecnología digital para su desarrollo en unos alumnos que han crecido bajo la influencia del uso de la red y de nuevas herramientas tecnológicas virtuales.

Aunque la innovación educativa no ha seguido el mismo ritmo de crecimiento que la tecnológica, en algunos contextos pueden llevarse a cabo metodologías innovadoras, como puede ser en los estudios superiores universitarios [5]. Los alumnos universitarios llevan generalmente en el aula un ordenador portátil o smartphone con acceso directo a la red. El empleo del smartphone y su inclusión en prácticas de juego en el aula puede suponer un primer paso en la generación de 'vivencias narrativas' [6].

En este sentido, la gamificación emerge como una herramienta de transformación educativa con el fin de adaptar el juego en la educación [7] que podemos utilizar para motivar el aprendizaje y potenciar el proceso de enseñanza en el aula [8]. Asimismo, el alumnado es cada vez más selectivo y crítico con la información que recibe en el aula, hecho que transforma estas experiencias de gamificación en un aliciente para su asistencia a clase [9].

Kahoot ${ }^{\circledR}$ es una plataforma gratuita que permite crear cuestionarios, encuestas y discusiones, y genera un feedback de los alumnos en tiempo real. Lo que se busca es fomentar un aprendizaje más activo e interactivo en el estudiante, promoviendo al mismo tiempo la participación en el aula. Esta herramienta ha sido estudiada previamente en el ámbito universitario por otros autores, en diversos programas de grado de diferentes áreas y distintas universidades $[10,11]$.

En este trabajo se presenta la valoración de un grupo de alumnos sobre el uso de Kahoot como herramienta educativa y como posible elemento motivador en el ámbito universitario.

\section{Sujetos y métodos}

La metodología planteada fue introducir esta técnica de aprendizaje, basada en preguntas tipo test, en las sesiones prácticas de la asignatura de 'Anatomía Humana II (Esplacnología)' que se ha impartido en el primer semestre del segundo curso del Grado de Medicina en la Universidad de Zaragoza durante el curso 2019-2020. En esta experiencia participaron los 84 alumnos matriculados en la asignatura. Para ello, se utilizó la aplicación Kahoot para dispositivos electrónicos (disponible de manera gratuita), que permitió la creación de cuestionarios online tipo Trivial ${ }^{\circledR}$ (basados en preguntas cortas con respuesta de elección múltiple), con el fin de evaluar los conocimientos adquiridos por el alumno en tiempo real.

El uso de este tipo aplicaciones es sencillo para el docente a la hora de realizar los cuestionarios online, y es quien marca las diferentes características de las cuestiones (puntuación otorgada, orden de las preguntas y tiempo de respuesta). Así mismo, también resulta sencillo para el alumno, pues tan solo necesita el game pin code que la propia aplicación genera de manera aleatoria, administrado por el docente en el momento de comenzar la actividad, y un nombre de usuario para entrar al juego (ya sea de manera individual o grupal). Las preguntas se proyectan en el aula y cada una de ellas muestra al respectivo ganador, acumulándose los puntos para ofrecer una clasificación final, como si se tratase de una competición.

Estos cuestionarios online permiten evaluar diversos contenidos educativos, gestionar el flujo y orden de las preguntas, y ver sus resultados en tiempo real en el aula de teoría o de prácticas, lo que permite fomentar una discusión con el alumnado y valorar las opciones de respuesta planteadas a cada una de las cuestiones. Del mismo modo, la aplicación proporciona después de cada pregunta una tabla clasificatoria que genera una motivación extra: la competitividad entre los participantes del estudio. Además, la aplicación permite almacenar los resultados en el perfil para poder visualizarlos posteriormente, e incluso obtener un resumen de ellos. En este resumen se recoge el cómputo de respuestas acertadas y fallidas, en general y por cada una de las preguntas, de forma que a pesar de haber realizado la revisión en el momento de la actividad, podamos reforzar aquellas cuestiones que han sido respondidas erróneamente por un mayor número de participantes.

Para la asignatura de esplacnología se plantearon un total de siete cuestionarios tipo test (uno por cada bloque en los que se divide la asignatura), ba- 
sados principalmente en imágenes, con cuatro opciones de respuesta, y relacionados con los contenidos de las sesiones prácticas, buscando reforzar el conocimiento y localización de las estructuras anatómicas estudiadas en la asignatura, tanto en preparaciones anatómicas (piezas naturales y maquetas) como en láminas ilustrativas.

Después de la realización de estos test, los alumnos contestaron dos encuestas, de elaboración propia y diseñadas específicamente para el desarrollo de este trabajo, que valoraban distintos aspectos relacionados con este método docente. La primera encuesta constaba de tres preguntas de respuesta afirmativa/negativa:

- ¿Habías utilizado anteriormente una herramienta de gamificación en el aula universitaria?

- ¿Te ha resultado útil el uso de Kahoot en esta asignatura?

- ¿Crees que es necesario el uso de este tipo de herramientas en la enseñanza universitaria?

La segunda encuesta constaba de tres cuestiones con cinco opciones de respuesta tipo Likert, indicando el grado de acuerdo o desacuerdo, desde 1 ('muy en desacuerdo') hasta 5 ('muy de acuerdo'):

- ¿Crees que el uso de tecnologías de la información y la comunicación), y en este caso la aplicación Kahoot, mejora el proceso de enseñanzaaprendizaje en la docencia práctica de la asignatura de esplacnología?

- ¿Crees que el uso de actividades de gamificación tipo Kahoot motivan al alumno en las sesiones prácticas?

- ¿Crees que la realización de las actividades programadas con la aplicación Kahoot, fomentando el uso de imágenes, te ha sido de ayuda para reforzar los conceptos aprendidos?

\section{Resultados}

El primer cuestionario de tres preguntas (respuesta afirmativa/negativa) fue respondido por el $71 \% \mathrm{del}$ alumnado $(n=62)$. De estas respuestas se pudo extraer que el $85 \%(n=53)$ no había utilizado estas herramientas de gamificación dentro del aula universitaria, mientras que el 100\% consideró de utilidad el uso de la plataforma Kahoot en el desarrollo práctico de la asignatura. Así mismo, el 91\% $(n=57)$ creyó que era necesario utilizar este tipo de herramientas en otras asignaturas dentro del Grado de Medicina.

Posteriormente, se realizaron las otras tres cuestiones (cinco opciones de respuesta según escala ti- po Likert). El resultado fue que el $88 \%(n=54)$ estaba de acuerdo o totalmente de acuerdo en que estas aplicaciones, utilizadas dentro del aula, mejoran el proceso de enseñanza-aprendizaje de la docencia práctica de la asignatura de esplacnología, mientras que un $12 \%(n=8)$ estaba en desacuerdo en el efecto de mejora de estas tecnologías en el proceso de aprendizaje.

El 89\% $(n=55)$ estaban de acuerdo o totalmente de acuerdo en que estas actividades mejoran su motivación en la docencia práctica de la asignatura, mientras que el $11 \%$ restante $(n=7)$ se mostraban neutrales, no estando ni de acuerdo ni en desacuerdo en el uso de estas actividades.

Respecto a la última pregunta, solamente el 3\% $(n=2)$ estaba en desacuerdo con el efecto de refuerzo de realizar estas actividades dentro del aula de prácticas, frente al 97\% $(n=60)$ que consideraba estar de acuerdo o totalmente de acuerdo con el efecto de refuerzo que ejercen este tipo de actividades.

\section{Discusión}

La gamificación, y en concreto la aplicación Kahoot, se perfila como una herramienta para un nuevo modelo de enseñanza dentro del aula universitaria [12], para implementar una serie de actividades digitales que permitan mantener la motivación de los 'nuevos' alumnos universitarios con ansia de tecnologías digitales, principalmente asociadas al uso de sus smartphones. Así mismo, recientes trabajos [13, 14] demuestran que realizar cuestionarios de elección múltiple, tipo test, basados en cuestiones clave, y si además se asocian con actividades relacionadas con el juego, conllevan una mejora en el proceso de enseñanza-aprendizaje en todos los niveles educativos $[6,15]$.

A día de hoy, son numerosos los docentes universitarios que siguen utilizando en sus métodos de enseñanza la clase magistral, entendiendo como tal una 'exposición unidireccional entre el docente y el alumno' $[15,16]$. Este tipo de exposición puede facilitar que se integren los conceptos de una manera más estructurada y permite reducir el contenido de la materia a los puntos más esenciales, pero sin embargo favorece la pasividad del alumno, que actúa como mero espectador en el aula al limitarse a tomar apuntes, lo que reduce la relación directa alumnoprofesor y puede llegar a disminuir la motivación del alumno en el desarrollo de la asignatura [16,17].

Con esta experiencia se puede corroborar que el uso de herramientas digitales en el aula a través del juego, y en concreto de la aplicación Kahoot, puede 
conseguir mejorar tanto la motivación como el proceso de enseñanza-aprendizaje de los alumnos. Sin embargo, aunque la gamificación mejora estos aspectos docentes, no todos los objetivos se consiguen con la misma eficacia utilizando cualquier método, lo que lleva a plantear como principio didáctico la diversidad metodológica para poder alcanzar todos los objetivos planteados, es decir, compatibilizar sistemas docentes más tradicionales con nuevas tecnologías.

\section{Bibliografía}

1. Luesma, MJ, Cantarero, I, Artal, JS, Abadía, AR. Píldoras educativas en la docencia de Anatomía e Histología Ocular. Experiencia de transferibilidad, 2019. Actas del V Congreso Internacional sobre Aprendizaje, Innovación y Cooperación (CINAIC). Madrid, 9-11 de octubre de 2019. URL: https:// doi.org/10.26754/cinaic.2019.0020. [11.11.2019].

2. Salinas-Ibáñez J. Innovación docente y uso de las TIC en la enseñanza universitaria. RUSC, Revista Universidad y Sociedad del Conocimiento 2004; 1: 1-16.

3. Ormart DE, Navés PFA. El uso de redes sociales como soporte educativo. CPU-e, Revista de Investigación Educativa 2014; 18: $172-81$.

4. Cerezo P. Generación Z vs millennials. Revista de Estudios de Juventud 2016; 114: 95-109.

5. Valenzuela R. Las redes sociales y su aplicación en la educación Revista Digital Universitaria 2013; 14: 1-14.

6. Rodríguez-Fernández L. Smartphones y aprendizaje: el uso de Kahoot en el aula universitaria. Revista Mediterránea de Comunicación 2017; 8: 181-90.

7. Montaner-Villalba S. Gamificación: cómo motivar a tu alumnado y mejorar el clima en el aula. Edutec, Revista Electrónica de Tecnología Educativa 2016; 55: a332.

8. Corchuelo-Rodriguez CA. Gamificación en educación superior experiencia innovadora para motivar estudiantes y dinamizar contenidos en el aula. Edutec, Revista Electrónica de Tecnología Educativa 2018; 63: 29-41.

9. Contreras-Espinosa RS, Eguía JL, eds. Experiencia de gamificacion en las aulas. Bellaterra: Institut de la ComunicacióUAB; 2017.

10. Fuertes A, García M, Castaño MA. López E, Zacares M, Cobos $\mathrm{M}$, et al. Uso de herramientas de respuesta de audiencia en la docencia presencial universitaria. Un primer contacto. Actas de las XXII JENUI. Almería: Universidad de Almería; 2016. p. 261-8.

11. Moya-Fuentes MM, Carrasco-Andrino MM, Jiménez-Pascua MA, Ramón-Martín A, Soler-García C, Vaello-López MT. $\mathrm{El}$ aprendizaje basado en juegos: experiencias docentes en la aplicación de la plataforma virtual Kahoot. Alacant: Universitat d'Alacant/Institut de Ciències de l'Educació; 2016. p. 1241-54.

12. Sempere-Ferré F. Kahoot como herramienta de autoevaluación en la universidad. IN-RED 2018: IV Congreso Nacional de Innovación Educativa y Docencia en Red. Valencia: Universitat Politècnica de València; 2018. p. 250-5.

13. Contreras RS, Eguía JL, eds. Gamificación en aulas universitarias. Bellaterra: Institut de la Comunicació-UAB; 2017.

14. Pujolà JT, Herrera-Jiménez FJ. Gamificación. In MuñozBasols J, Gironzetti E, Lacorte M, eds. The Routledge handbook of Spanish language teaching. New York: Taylor \& Francis Books; 2018. p. 583-95.

15. Valcárcel MV. Presentación y explicación de los contenidos: la clase magistral. Plan de formación inicial del profesorado de la Universidad de Murcia. Murcia: Universidad de Murcia; 2014

16. Vargas-Duarte J. Clase magistral. Revista Signos 1998; 31: 175-81.

17. Gerardo-Mateu J. La interacción en la clase magistral. Porta Linguarum: Revista Internacional de Didáctica de Las Lenguas Extranjeras 2005; 4: 201-2. 